\section{Non-invasive monitoring of hepatic steatosis via acoustic structure quantification of ultrasonography with MR spectroscopy as the reference standard}

\author{
Dong Ho Lee', Jae Young Lee ${ }^{1,2}$, Min Sun Park ${ }^{3}$, Joon Koo Han ${ }^{1,2}$ \\ ${ }^{1}$ Department of Radiology, ${ }^{2}$ Institute of Radiation Medicine, ${ }^{3}$ Department of Family Medicine, \\ Seoul National University Hospital, Seoul, Korea
}

Purpose: The purpose of this study was to prospectively evaluate whether monitoring hepatic steatosis by ultrasonography with acoustic structure quantification (ASQ) is feasible, using magnetic resonance spectroscopy (MRS) as the reference standard.

Methods: Thirty-six patients with suspected fatty liver disease underwent both untrasonography with ASQ and MRS on the same day. After a mean follow-up period of $11.4 \pm 2.5$ months, followup ultrasonography with ASQ and MRS were performed on 27 patients to evaluate whether hepatic steatosis had improved. The focal disturbance (FD) ratio, as calculated using ASQ, and the hepatic fat fraction (HFF), estimated by MRS, were obtained at both initial and follow-up examinations. Pearson correlation coefficients were calculated to assess the correlations between ordinal values.

Results: The FD ratio showed a strong, negative linear correlation with the HFF after logarithmic transformation of both variables from the initial examinations of 36 patients $(\rho=-0.888$, $\mathrm{P}<0.001)$ and the follow-up examinations of 27 patients $(\rho=-0.920, P<0.001)$. There was also a significant, negative linear correlation between the change in the logarithm of the FD ratio and the change in the logarithm of the HFF by MRS over the follow-up period $(\rho=-0.645, P<0.001)$. In 16 patients with an increased FD ratio on follow-up, the HFF on follow-up MRS significantly decreased, and high-density lipoprotein levels significantly increased, whereas low-density lipoprotein levels tended to decrease.

Conclusion: The FD ratio was significantly correlated with the HFF at both the initial and followup examinations, and there was also a significant correlation between changes in the FD ratio and changes in the HFF over the follow-up period.

Keywords: Hepatic steatosis; Acoustic structure quantification; Magnetic resonance spectroscopy; Monitoring hepatic steatosis

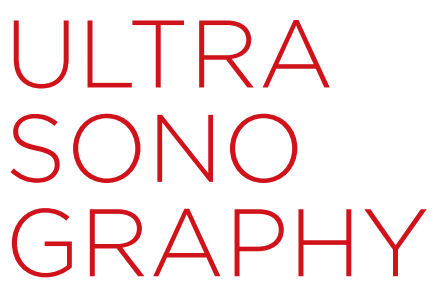

ORIGINAL ARTICLE

https://doi.org/10.14366/usg. 19002 pISSN: 2288-5919 • elSSN: 2288-5943 Ultrasonography 2020;39:70-78

Received: January 6, 2019

Revised: June 20, 2019

Accepted: June 25, 2019

Correspondence to: Jae Young Lee, MD, Department of Radiology, Seoul National University Hospital, 101 Daehak-ro, Jongno-gu, Seoul 03080, Korea

Tel. +82-2-2072-3073

Fax. +82-2-743-6385

E-mail: leejy4u@snu.ac.kr

This is an Open Access article distributed under the terms of the Creative Commons Attribution NonCommercial License (http://creativecommons.org/ licenses/by-nc/4.0/) which permits unrestricted noncommercial use, distribution, and reproduction in any medium, provided the original work is properly cited.

Copyright $(2020$ Korean Society of Ultrasound in Medicine (KSUM)

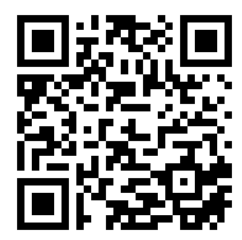

How to cite this article:

Lee DH, Lee JY, Park MS, Han JK. Non-invasive monitoring of hepatic steatosis via acoustic structure quantification of ultrasonography with MR spectroscopy as the reference standard. Ultrasonography. 2020 Jan;39(1):7078. 


\section{Introduction}

Hepatic steatosis is defined as the excessive and abnormal accumulation of intracellular lipids, primarily in the form of triglycerides. Clinically, the most common type of hepatic steatosis is nonalcoholic fatty liver disease (NAFLD), which includes a broad spectrum of diseases, ranging from simple steatosis to nonalcoholic steatohepatitis (NASH). In addition, NASH can progress to fibrosis/ cirrhosis, and end-stage liver disease requiring liver transplantation can eventually develop when it is not appropriately managed [1-4]. Currently, NAFLD is the most common cause of chronic liver disease in Western countries, with a prevalence of approximately $30 \%$ in the general population, and the incidence of NAFLD is known to be increasing $[5,6]$. Therefore, NAFLD is a major emerging health problem, and the early detection and quantitative assessment of NAFLD are of crucial importance for preventing progression to advanced stages, such as cirrhosis.

Traditionally, liver biopsy has been considered the gold standard for the diagnosis and quantification of hepatic steatosis [7]. However, due to its invasive nature and procedure-related complications and mortality, repeated biopsies for monitoring NAFLD should rarely be performed. Therefore, hydrogen-1 $\left({ }^{1} \mathrm{H}\right)$ magnetic resonance spectroscopy (MRS), which is an accurate and reproducible method for assessing hepatic steatosis, has been used as an alternative, non-invasive standard of reference $[8,9]$. In addition, ${ }^{1} \mathrm{H}$-MRS can be used to monitor hepatic steatosis [10]. However, its high cost and the limited availability of MRS compared to other non-invasive imaging modalities, such as ultrasonography (US), are disadvantages.

Recently, acoustic structure quantification (ASQ), which is a newly developed quantitative analysis method for US reported by Toshiba Medical Systems (Otawara, Japan), has been introduced to clinical practice. ASQ is based on comparisons between theoretical and real echo amplitude distributions [11-13], and the theoretical echo amplitude distribution of the liver region is considered using the Rayleigh distribution function, based on the assumption that the speckle pattern is generated by only ultrasound beam interference from very small scattering objects that are located closer than the ultrasound beam wavelength [11-13]. However, the real echo amplitude distribution of the liver parenchyma does not actually fit a Rayleigh distribution because of the presence of small structures, such as the hepatic vessel walls, which scatter the ultrasound beam and cause heterogeneity in the echo amplitude [11-13]. By comparing the theoretical echo amplitude distribution to a real distribution, ASQ can provide quantitative information regarding changes of parenchymal echotexture in diffuse liver diseases, such as steatosis. According to the results of recent studies, the focal disturbance $(F D)$ ratio calculated using the ASQ technique was significantly associated with the degree of hepatic steatosis assessed by histopathology, and there was a strong, negative correlation between the FD ratio and the estimated fat fraction assessed by MRS $[12,13]$. Given these findings, the FD ratio calculated using the ASQ technique could be used to monitor hepatic steatosis. However, the utility of the ASQ technique as a tool for monitoring hepatic steatosis has not been evaluated. Therefore, the purpose of our study was to prospectively evaluate whether monitoring hepatic steatosis using US with ASQ was feasible in a fatty liver disease patient cohort, using ${ }^{1} \mathrm{H}$-MRS as the reference standard.

\section{Materials and Methods}

\section{Study Population}

The institutional review board of our hospital approved this prospective study, and written informed consent was obtained from all enrolled patients. From November 2014 to April 2015, we enrolled 36 patients who visited our institution for suspected or verified hepatic steatosis and received routine US examinations. The electronic medical records and past medical histories of all patients were reviewed to confirm that they did not have any past medical histories or clinical symptoms or signs of liver disease or systemic or malignant diseases. Laboratory findings, including liver function tests obtained within 3 months before the imaging examinations, were also checked in all of the patients. All patients underwent both US examinations that used the ASQ technique and ${ }^{1} \mathrm{H}-\mathrm{MRS}$ examinations on the same day. After the initial imaging examination, lifestyle interventions were conducted in all of the enrolled patients, with dietary counseling aimed to reduce body weight. The patients were also instructed to perform at least 3 hours of moderate physical activity per week, and aerobic endurance exercise, including walking and swimming, was encouraged [14-17]. All of the enrolled patients were seen by a physician (M.S.P.) on a regular basis. After a mean and median follow-up of $11.4 \pm 2.5$ (standard deviation) months and 12.0 months (range, 6 to 15 months), respectively, follow-up US examinations using ASQ, ${ }^{1} \mathrm{H}$-MRS examinations and laboratory tests, including liver function tests, were performed in 27 of the 36 patients to evaluate whether hepatic steatosis had improved; follow-up US and MRS examinations were done on the same day. Nine patients (9 of 36, 25.0\%) refused to undergo followup imaging examinations. The characteristics of the patient cohort are summarized in Table 1.

\section{US Examinations}

All of the US examinations were performed by one of the two authors (J.Y.L. and D.H.L. with 20 and 9 years of experience, 
respectively, in liver US imaging) using a US scanner (Aplio XG, Toshiba Medical Systems) with a $5-\mathrm{MHz}$ convex transducer. All patients fasted for at least 6 hours before the imaging examinations. The US examinations were done with patients in the supine position with their right arm extended above their head to stretch the intercostal muscle for a better sonic window; the same patient position was used in both the initial and follow-up examinations. First, a B-mode US examination of the liver was performed, including transverse and sagittal images, to evaluate the presence of any possible focal liver lesions. Then, US images in ASQ mode were acquired 5 times each from the right intercostal view and the right subcostal view. All five regions of interest (ROIs) were placed in the same intercostal and right subcostal views. The display depth and transmit focus were set to $10 \mathrm{~cm}$ and $6 \mathrm{~cm}$, respectively. The ROls were as large as possible and were placed on the liver parenchyma; care was taken to avoid large hepatic vessels or artifacts. The value of the FD ratio was calculated by the system and was displayed

Table 1. Baseline characteristics of the 36 patients with suspected fatty liver disease

\begin{tabular}{|c|c|}
\hline Characteristic & Value \\
\hline Age (yr) & $50.4 \pm 13.3(20-71)$ \\
\hline \multicolumn{2}{|l|}{ Sex } \\
\hline Male & $21(58.3)$ \\
\hline Female & $15(41.7)$ \\
\hline \multicolumn{2}{|l|}{ Diabetes } \\
\hline Yes & $10(27.8)$ \\
\hline No & $26(72.2)$ \\
\hline \multicolumn{2}{|l|}{ Hypertension } \\
\hline Yes & $17(47.2)$ \\
\hline No & $19(52.8)$ \\
\hline \multicolumn{2}{|l|}{ Hyperlipidemia } \\
\hline Yes & $21(58.3)$ \\
\hline No & $15(41.7)$ \\
\hline Serum albumin (mg/dL) & $4.5 \pm 0.2(4.1-4.9)$ \\
\hline Total bilirubin level (mg/dL) & $0.90 \pm 0.31(0.6-1.7)$ \\
\hline Alanine aminotransferase (IU/dL) & $49.6 \pm 43.2(14-220)$ \\
\hline Body weight (kg) & $80.4 \pm 18.6(55-124)$ \\
\hline Body mass index $\left(\mathrm{kg} / \mathrm{m}^{2}\right)$ & $28.4 \pm 4.6(22-39)$ \\
\hline Total cholesterol (mg/dL) & $194.2 \pm 38.5(125-267)$ \\
\hline Triglycerides (mg/dL) & $164.8 \pm 92.3(45-433)$ \\
\hline Low-density lipoprotein (mg/dL) & $124.3 \pm 44.3(54-216)$ \\
\hline High-density lipoprotein (mg/dL) & $49.9 \pm 12.5(32-90)$ \\
\hline Estimated hepatic fat fraction by MRS (\%) & $16.7 \pm 9.7(1.8-39.3)$ \\
\hline
\end{tabular}

Values are presented as mean $\pm \mathrm{SD}$ (range) or number (\%).

MRS, magnetic resonance spectroscopy; SD, standard deviation. on the monitor (Fig. 1). The mean FD ratio of five measurements was calculated for each patient and recorded for further analysis. Detailed methods and a description of the ASQ technique and the calculation of the FD ratio are provided in the appendix.

\section{${ }^{1} \mathrm{H}-\mathrm{MRS}$}

All ${ }^{1} \mathrm{H}$-MRS examinations were performed on a clinical 3.0-T MR scanner (Trio A Tim System, Siemens Healthcare, Erlangen, Germany) with a 32-channel phased-array surface coil on the same day of the US examinations. First, sagittal, coronal, and axial sections that covered the whole liver were obtained to position the spectroscopy acquisition voxel. Then, a signal voxel measuring $3 \times 3 \times 3 \mathrm{~cm}$ was placed in the right hepatic lobe dome area of Couinaud segment VII or VIII, with care taken to avoid large blood vessels or bile ducts and liver edges. For the fat fraction spectroscopy measurements, a modified stimulated-echo acquisition sequence (echo time [msec]/ repetition time [msec]; 12, 24, 36, 48 and 72/3,000) was employed within the single voxel. Each acquisition was performed during a single breath hold with a scan time of 15 seconds. T2 correction for both the water and lipids was performed to calculate the hepatic fat fraction (HFF), and each $\mathrm{T} 2$ value of water and fat was calculated separately. By using an exponential fit of the points acquired at five different echoes by automatic fitting of water $(4.7 \mathrm{ppm})$ and the major fat peak of methylene (1.3 ppm), extrapolation of fat and water integrals for an echo time of 0 msec was performed for fat quantification $[8,18]$. The calculated HFF was automatically displayed as a percentage in Distal Imaging and Communications in Medicine format and was recorded for further analysis.

\section{Statistical Analysis}

The estimated HFF by MRS and the FD ratio calculated using the ASQ technique are expressed as the mean \pm standard deviation throughout the results. We used Pearson correlation coefficients to assess the correlation between the FD ratio and the HFF. After the initial examination, 27 of the 36 patients underwent follow-up US examinations using the ASQ technique and ${ }^{1} \mathrm{H}$-MRS examinations, and the changes in the FD ratio, as well as the estimated HFF by MRS, between the initial and follow-up examinations were calculated. Using the changes in FD ratio calculated by ASQ over the follow-up period, patients were classified into two groups depending on whether their FD ratio increased or decreased during follow-up. Differences in the estimated HFF on MRS, as well as in other characteristics of patients, between the initial and followup examinations were assessed by group using the Wilcoxon signed-rank test. All statistical analyses were performed using SPSS software version 21 (IBM Corp., Armonk, NY, USA), and MedCalc software version 12.1.0.0 (MedCalc Software, Mariakerke, Belgium). 


\section{Results}

FD Ratio and Estimated HFF at the Initial Examinations

The mean estimated HFF at the initial MRS examinations of all 36 patients was $16.7 \% \pm 9.7 \%$ (range, $1.8 \%$ to $39.3 \%$ ), and the mean FD ratio calculated using the ASQ technique at the initial US examinations was $0.07 \pm 0.10$ (range, 0.02 to 0.56 ). The correlation coefficient $(\rho)$ between the FD ratio calculated using the ASQ technique and the estimated HFF by MRS was $-0.526(95 \%$

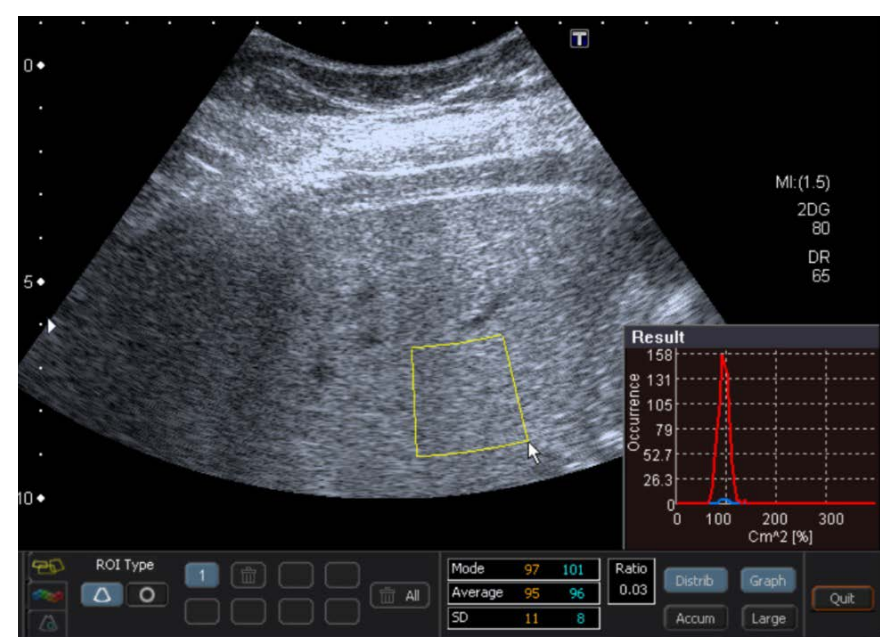

A

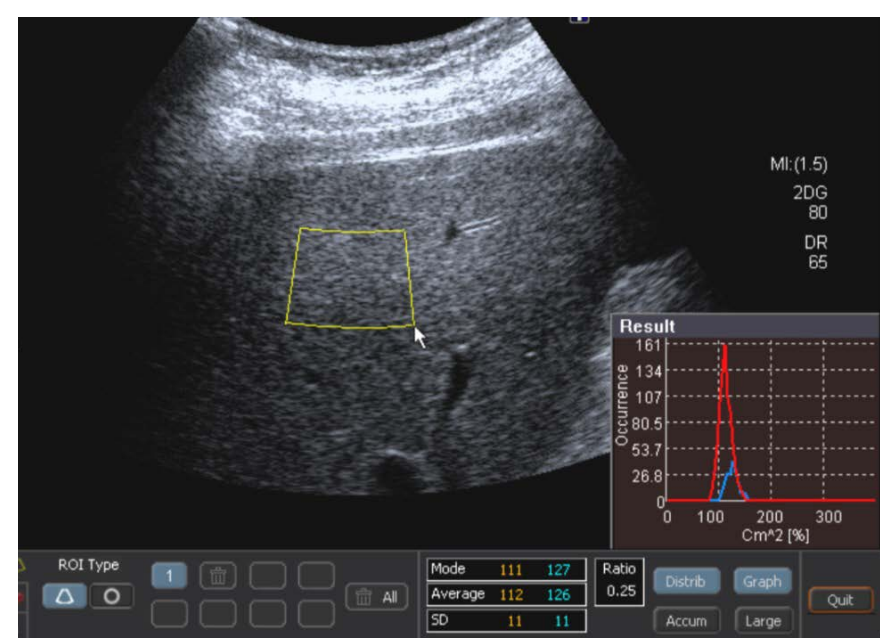

C

Fig. 1. Initial and follow-up ultrasonography (US) examinations using the acoustic structure quantification (ASQ) technique and magnetic resonance spectroscopy (MRS) examinations in a 29-year-old man with fatty liver.

A. Upon initial examination, the patient's body weight was $115 \mathrm{~kg}$, and he had a body mass index (BMI) of $36 \mathrm{~kg} / \mathrm{m}^{2}$. On an ASQ-mode US image, the region of interest was placed on the liver parenchyma, and care was taken to avoid large hepatic vessels or artifacts. The focal disturbance (FD) ratio calculated by ASQ in this patient was 0.03 . B. The estimated hepatic fat fraction (HFF) according to the initial MRS examination of this patient was $10.14 \%$. C. During the 10-month follow-up period, the patient lost $21 \mathrm{~kg}$ of body weight, and his BMI at the follow-up examination was $30 \mathrm{~kg} / \mathrm{m}^{2}$. The FD ratio calculated by ASQ for the follow-up examination was 0.25 . D. The estimated HFF for the follow-up MRS examination was $2.93 \%$. Cl, confidence interval; TE, echo time.

e-ultrasonography.org

Ultrasonography 39(1), January 2020 
examinations were performed on 27 patients, and the mean estimated HFF calculated at the follow-up MRS examinations was $14.0 \% \pm 9.3 \%$ (range, $1.0 \%$ to $32.0 \%$ ). The mean FD ratio calculated using the ASQ technique at the follow-up US examinations was $0.10 \pm 0.11$ (range, 0.02 to 0.53 ). The correlation coefficient $(\rho)$ between the FD ratio calculated using the ASQ technique for the follow-up examinations and the estimated HFF according to the follow-up MRS examinations in these 27 patients was -0.637 $\left(95 \% \mathrm{Cl}_{1}-0.338\right.$ to -0.819$)$. A scatter plot of the estimated HFF and the FD ratio obtained from the follow-up examinations also showed an exponential relationship. There was a strong, negative linear correlation between the logarithm of the FD ratio obtained at the follow-up US examinations and the ASQ technique and the logarithm of the HFF of the follow-up MRS examinations, and the correlation coefficient $(\rho)$ was $-0.920(95 \% \mathrm{Cl},-0.831$ to -0.963 ; $\mathrm{P}<0.001$ ) (Fig. 3).

Changes in the FD Ratio over the Follow-up Period and Correlation with the HFF

Among the 27 patients who underwent follow-up examination, the FD ratio calculated by ASQ was higher at the follow-up US examination than at the initial US examination in 16 patients and lower in 11 patients. The correlation coefficient $(\rho)$ between the

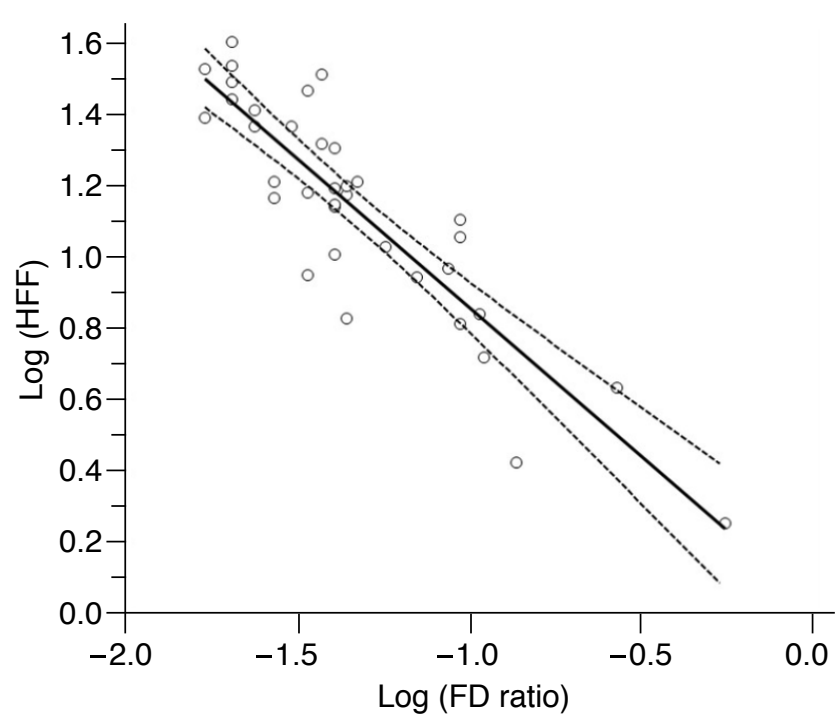

Fig. 2. Correlation between the focal disturbance (FD) ratio and the hepatic fat fraction (HFF) at the initial examinations of all 36 patients. Regression plot showing a strong negative linear correlation between the FD ratio and the HFF after the logarithmic transformation of both variables; the correlation coefficient was $-0.888(P<0.001)$. The solid line indicates the regression line, and the lines with small dots are the $95 \%$ confidence intervals. change in the FD ratio and the change in the estimated HFF was $-0.553(95 \% \mathrm{Cl},-0.219$ to -0.771$)$. A scatter plot of the change in the estimated HFF and the change in the FD ratio over the followup period also showed an exponential relationship. There was also a significant, negative linear correlation between the change in the logarithm of the FD ratio over the follow-up period and the change in the logarithm of the HFF over the follow-up period by MRS, and the correlation coefficient $(\rho)$ was $-0.645(95 \% \mathrm{Cl},-0.351$ to $-0.823 ; \mathrm{P}<0.001$ ) (Fig. 4).

\section{Changes in Patient Characteristics According to Changes in the FD Ratio}

Changes in patient characteristics over the follow-up period according to changes in the FD ratio calculated using ASQ are summarized in Table 2. Body weight decreased over the course of follow-up, but a statistically significant difference was not found. The estimated HFF on follow-up MRS significantly decreased in the 16 patients with an increased FD ratio at follow-up $(P=0.003)$. In addition, high-density lipoprotein (HDL) levels significantly increased over follow-up in the 16 patients with an increased FD ratio $(P=0.035)$. There was a tendency for low-density lipoprotein $(L D L)$ levels to decrease during follow-up in the 16 patients with increased $F D$ ratio $(P=0.084)$. In the 11 patients with a decreased FD ratio, the

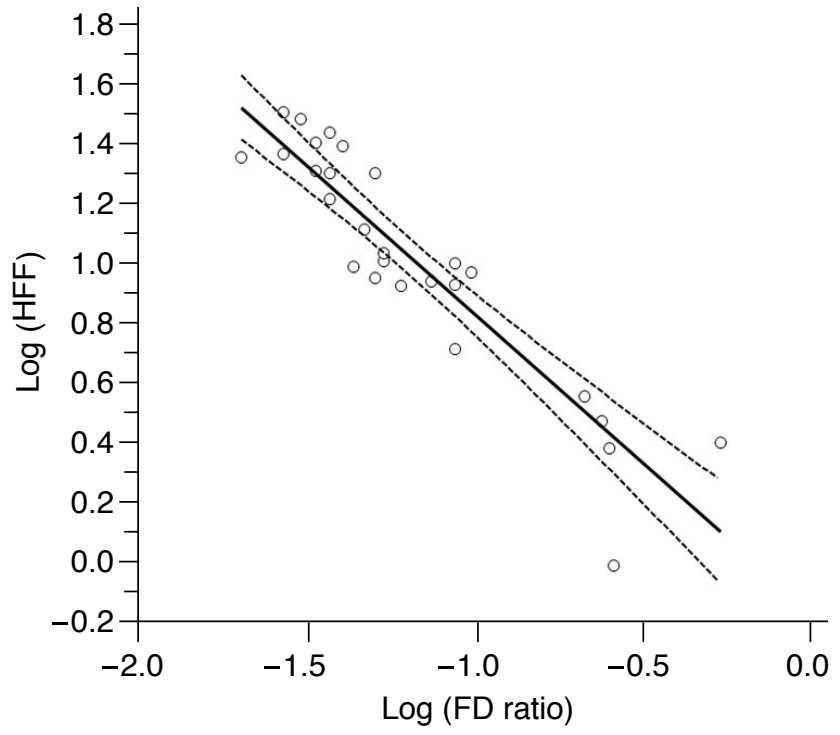

Fig. 3. Correlation between the focal disturbance (FD) ratio and the hepatic fat fraction (HFF) at the follow-up examinations of 27 patients. Regression plot showing a strong negative linear correlation between the FD ratio and the HFF after the logarithmic transformation of both variables; the correlation coefficient was $-0.920(P<0.001)$. The solid line indicates the regression line, and the lines with small dots are the $95 \%$ confidence intervals. 
Table 2. Changes in parameters in the 27 patients who underwent follow-up examinations according to the change in the FD ratio calculated by the ASQ technique at the follow-up examination

\begin{tabular}{|c|c|c|c|c|c|c|}
\hline & \multicolumn{3}{|c|}{ Increased FD ratio on follow-up $(n=16)$} & \multicolumn{3}{|c|}{ Decreased FD ratio on follow-up $(n=11)$} \\
\hline & Initial & Follow-up & P-value ${ }^{a)}$ & Initial & Follow-up & P-value $e^{a)}$ \\
\hline Body weight (kg) & $82.8 \pm 16.9$ & $79.8 \pm 13.5$ & 0.158 & $76.1 \pm 16.8$ & $74.2 \pm 16.7$ & 0.260 \\
\hline Albumin (mg/dL) & $4.6 \pm 0.2$ & $4.5 \pm 0.2$ & 0.287 & $4.5 \pm 0.2$ & $4.5 \pm 0.3$ & 0.887 \\
\hline Total bilirubin (mg/dL) & $1.08 \pm 0.37$ & $0.99 \pm 0.25$ & 0.216 & $0.84 \pm 0.23$ & $0.68 \pm 0.22$ & 0.151 \\
\hline $\operatorname{ALT}(\mathrm{IU} / \mathrm{dL})$ & $59.8 \pm 56.5$ & $47.8 \pm 32.9$ & 0.780 & $34.6 \pm 16.8$ & $30.0 \pm 12.6$ & 0.406 \\
\hline Total cholesterol (mg/dL) & $188.5 \pm 40.9$ & $185.9 \pm 33.2$ & 0.851 & $184.7 \pm 36.2$ & $158.1 \pm 33.8$ & 0.203 \\
\hline Triglycerides (mg/dL) & $146.3 \pm 71.9$ & $136.4 \pm 44.4$ & 0.753 & $163.6 \pm 82.0$ & $145.6 \pm 72.0$ & 0.241 \\
\hline LDL (mg/dL) & $128.6 \pm 44.4$ & $109.8 \pm 35.7$ & 0.084 & $108.3 \pm 38.4$ & $100.9 \pm 37.3$ & 0.241 \\
\hline $\mathrm{HDL}(\mathrm{mg} / \mathrm{dL})$ & $47.1 \pm 8.4$ & $50.7 \pm 9.8$ & 0.035 & $48.1 \pm 9.7$ & $49.6 \pm 12.4$ & 0.384 \\
\hline Fat fraction on MRS (\%) & $17.6 \pm 11.8$ & $13.3 \pm 8.8$ & 0.003 & $12.4 \pm 8.6$ & $15.0 \pm 10.3$ & 0.248 \\
\hline
\end{tabular}

FD ratio, focal disturbance ratio; ASQ, acoustic structure quantification; ALT, alanine aminotransferase; LDL, low-density lipoprotein; HDL, high-density lipoprotein; MRS, magnetic resonance spectroscopy.

${ }^{a}$ The P-value was determined using the Wilcoxon signed-rank test.

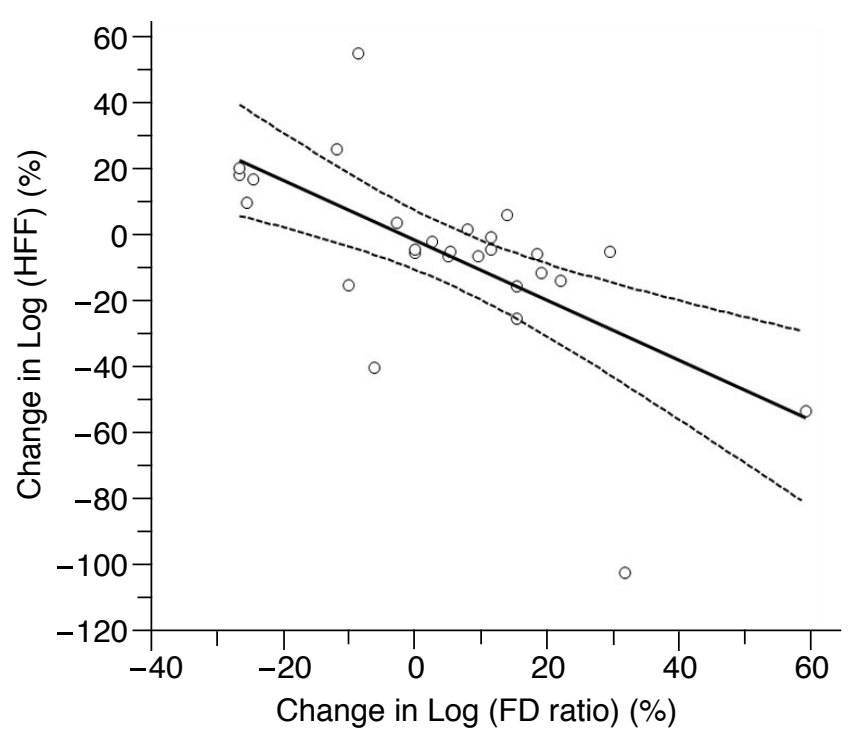

Fig. 4. Correlation between changes in the focal disturbance (FD) ratio and the hepatic fat fraction (HFF) over follow-up in the 27 patients who underwent follow-up examinations. Regression plot showing a significant negative linear correlation between changes in the logarithm of the FD ratio and the logarithm of the HFF; the correlation coefficient was $-0.645(P<0.001)$. The solid line indicates the regression line, and the lines with small dots are the $95 \%$ confidence intervals.

estimated HFF increased on follow-up MRS, without a statistically significant difference $(\mathrm{P}=0.248)$. Other patient characteristics were not significantly different at the follow-up examinations in the 11 patients with a decreased FD ratio.

\section{Discussion}

In our study, the FD ratio calculated using the ASQ technique showed a significant, negative correlation with the estimated HFF by MRS at both the initial and follow-up examinations, corresponding closely to the findings of a previous study by Son et al. [13]. In addition, there was a significant correlation between the change in the FD ratio over the follow-up period and the change in the HFF over the follow-up period by MRS. An increased FD ratio on followup US with the ASQ technique compared to the initial examination was significantly associated with a decreased HFF on follow-up MRS. Moreover, HDL levels significantly increased and LDL levels tended to decrease in the 16 patients with an increased FD ratio on the follow-up examination, which could indicate improvement of their lipid panels.

Because NAFLD encompasses a broad spectrum, ranging from simple steatosis to cirrhosis, and can progress into more advanced stages during the disease course, the quantitative assessment and monitoring of this disease are important for patient management. US is a non-invasive imaging modality for assessing liver disease with a relatively lower cost compared to magnetic resonance imaging, and it can be performed repeatedly during the disease course with no risk to the patient. Given this finding, US could potentially be a good imaging modality for monitoring fatty liver disease. When the echogenicity of the liver parenchyma increases compared to that of the renal cortical parenchyma on B-mode US, it is usually considered to indicate the presence of hepatic steatosis. However, the assessment of hepatic parenchymal echogenicity by B-mode US is subjective in nature. In addition, quantifying the 
degree of hepatic steatosis is only barely possible by using B-mode US images alone. To overcome these drawbacks of B-mode US, several amendments, including the hepatorenal ratio, have been suggested for the quantitative assessment of hepatic steatosis, with relatively good performance [19-21]. However, although Mancini et al. [22] reported that the hepatorenal ratio showed an excellent correlation with the degree of steatosis evaluated by MRS, whether this ratio has sufficient diagnostic performance for assessing hepatic steatosis or could be used an alternative tool to MRS remains questionable [13]. In addition, other studies have reported that additional processes, such as standardization or use of artificial neural networks, would be needed for the hepatorenal ratio to attain a sufficiently high diagnostic performance for the quantitative assessment of hepatic steatosis [21,23].

In the ASQ technique, the theoretical echo amplitude distribution, which is believed to be a function of the Rayleigh distribution, is compared to a real echo amplitude distribution, which does not actually fit a Rayleigh distribution, mainly due to the presence of small structures, including hepatic vessels, that scatter the US waves [11-13]. As the degree of hepatic steatosis progresses, the small structures in the liver, including small vessels, are blurred with the increased hepatic parenchymal echogenicity; in other words, hepatic parenchymal echogenicity might become more homogeneous. In such cases, the real echo amplitude distribution of the liver parenchyma can become similar to the theoretical echo amplitude distribution (i.e., the Rayleigh distribution) [13]. Comparing the theoretical echo amplitude distribution to a real distribution, the FD ratio calculated using the ASQ technique could provide quantitative information regarding the hepatic parenchymal echo texture changes that occur during the progression of hepatic steatosis. Our study results clearly showed a significant correlation between the FD ratio calculated by the ASQ technique and the estimated HFF by MRS at both the initial and follow-up examinations. In addition, the change in the FD ratio over the follow-up period was significantly correlated with the change in the estimated HFF by MRS, and an increased FD ratio on follow-up examination was significantly associated with a decreased HFF on follow-up MRS. Considering this result, the FD ratio calculated using the ASQ technique could potentially be used to quantitatively monitor fatty liver disease.

Currently, NAFLD is considered to be a hepatic manifestation of metabolic syndrome, which is characterized by the concomitant existence of type 2 diabetes, hypertension, dyslipidemia, and atherosclerotic cardiovascular disease [24]. In addition, Sinn et al. [25] recently reported that NAFLD was significantly associated with the development of coronary calcified plaque, independent of cardiovascular and metabolic risk factors. Therefore, accurate evaluation and monitoring of NAFLD could be quite important for reducing the potential mortality and morbidity from cardiovascular disease. In this regard, quantitative assessment of hepatic steatosis using US with the ASQ technique would be helpful in the management of NAFLD patients, as our study results showed that the FD ratio calculated using the ASQ technique was significantly correlated with the estimated HFF on MRS, which is a reference standard for non-invasive methods of diagnosing NAFLD. In addition, an increased FD ratio at the follow-up examination was also associated with significantly increased HDL levels and a tendency for decreased LDL levels, potentially indicating improvements in the lipid panel. Considering our study results, US examinations using the ASQ technique could be a promising monitoring method not only for hepatic steatosis, but also for metabolic syndrome.

Our study had some limitations. First, in our study, ${ }^{1} H-M R S$ examinations, instead of histologic examinations, were used as the reference standard to quantitatively assess hepatic steatosis. Therefore, the relationship between the FD ratio calculated using the ASQ technique and histologic changes could not be evaluated in our study. However, many clinical trials use ${ }^{1} \mathrm{H}$-MRS as the reference standard to assess the degree of hepatic steatosis due to its accuracy and its quantitative and noninvasive nature. Second, the number of patients was relatively small, especially those undergoing follow-up examinations. Third, we did not perform liver biopsy or elastography. Therefore, we could not assess the effect of fibrosis or inflammation on the FD ratio. In addition, according to the results of the recent animal study conducted by Lee et al. [26], the degrees of both steatosis and fibrosis were significant factors affecting the FD ratio calculated by ASQ, although the correlation coefficient for steatosis was higher than that for fibrosis. Indeed, it is unclear whether the FD ratio calculated using the ASQ technique can provide good diagnostic performance for quantitatively assessing hepatic steatosis in patients with both steatosis and inflammation/fibrosis. Further studies with prospective designs and large numbers of patients with various stages of fatty liver disease, including steatosis and cirrhosis, are warranted to address this issue. Fourth, as the MRS signal voxel was placed on the right liver dome, where accurate US evaluation would be difficult, there could have been some differences between the area evaluated using the ASQ technique and the area assessed by MRS; therefore, there may be some limitations regarding the accuracy of the correlation between the FD ratio calculated by ASQ and the estimated HFF by MRS.

In conclusion, the FD ratio calculated using the ASQ technique was significantly correlated with the estimated HFF by MRS at both the initial and follow-up examinations, and there was a significant correlation between the changes in the FD ratio and the changes in the HFF over the follow-up period, suggesting that the FD ratio can be used to quantitatively monitor fatty liver disease. 
ORCID: Dong Ho Lee: https://orcid.org/0000-0001-8983-851X; Jae Young Lee: https://orcid.org/0000-0001-6946-6042; Min Sun Park: https://orcid.org/0000-00016120-8356; Joon Koo Han: https://orcid.org/0000-0001-5916-5545

\section{Conflict of Interest}

No potential conflict of interest relevant to this article was reported.

\section{Author Contributions}

Conceptualization: Lee JY. Data acquisition: Lee DH, Lee JY, Park MS. Data analysis or interpretation: Lee DH. Drafting of the manuscript: Lee DH. Critical revision of the manuscript: Park MS, Han JK, Lee JY. Approval of the final version of the manuscript: all authors.

\section{References}

1. Sanyal AJ; American Gastroenterological Association. AGA technical review on nonalcoholic fatty liver disease. Gastroenterology 2002;123:1705-1725.

2. Kleiner DE, Brunt EM, Van Natta M, Behling C, Contos MJ, Cummings $\mathrm{OW}$, et al. Design and validation of a histological scoring system for nonalcoholic fatty liver disease. Hepatology 2005;41:1313-1321.

3. Marsman HA, van Werven JR, Nederveen AJ, Ten Kate FJ, Heger $\mathrm{M}$, Stoker J, et al. Noninvasive quantification of hepatic steatosis in rats using 3.0 T 1H-magnetic resonance spectroscopy. J Magn Reson Imaging 2010;32:148-154.

4. Oh H, Jun DW, Saeed WK, Nguyen MH. Non-alcoholic fatty liver diseases: update on the challenge of diagnosis and treatment. Clin Mol Hepatol 2016;22:327-335.

5. Bellentani S, Bedogni G, Miglioli L, Tiribelli C. The epidemiology of fatty liver. Eur J Gastroenterol Hepatol 2004;16:1087-1093.

6. Browning JD, Szczepaniak LS, Dobbins R, Nuremberg P, Horton $J D$, Cohen JC, et al. Prevalence of hepatic steatosis in an urban population in the United States: impact of ethnicity. Hepatology 2004;40:1387-1395.

7. Bravo AA, Sheth SG, Chopra S. Liver biopsy. N Engl J Med 2001;344:495-500.

8. Pineda N, Sharma P, Xu Q, Hu X, Vos M, Martin DR. Measurement of hepatic lipid: high-speed T2-corrected multiecho acquisition at $1 \mathrm{H}$ MR spectroscopy: a rapid and accurate technique. Radiology 2009:252:568-576.

9. Lee $\mathrm{DH}$. Imaging evaluation of non-alcoholic fatty liver disease: focused on quantification. Clin Mol Hepatol 2017;23:290-301.

10. Cowin GJ, Jonsson JR, Bauer JD, Ash S, Ali A, Osland EJ, et al. Magnetic resonance imaging and spectroscopy for monitoring liver steatosis. J Magn Reson Imaging 2008;28:937-945.

11. Toyoda H, Kumada T, Kamiyama N, Shiraki K, Takase K, Yamaguchi $\mathrm{T}$, et al. B-mode ultrasound with algorithm based on statistical analysis of signals: evaluation of liver fibrosis in patients with chronic hepatitis C. AJR Am J Roentgenol 2009;193:1037-1043.

12. Kuroda H, Kakisaka K, Kamiyama N, Oikawa T, Onodera M, Sawara $K$, et al. Non-invasive determination of hepatic steatosis by acoustic structure quantification from ultrasound echo amplitude. World J Gastroenterol 2012;18:3889-3895.

13. Son JY, Lee JY, Yi NJ, Lee KW, Suh KS, Kim KG, et al. Hepatic steatosis: assessment with acoustic structure quantification of US imaging. Radiology 2016;278:257-264.

14. Hickman IJ, Jonsson JR, Prins JB, Ash S, Purdie DM, Clouston AD, et al. Modest weight loss and physical activity in overweight patients with chronic liver disease results in sustained improvements in alanine aminotransferase, fasting insulin, and quality of life. Gut 2004;53:413-419.

15. Suzuki A, Lindor K, St Saver J, Lymp J, Mendes F, Muto A, et al. Effect of changes on body weight and lifestyle in nonalcoholic fatty liver disease. J Hepatol 2005;43:1060-1066.

16. Kantartzis K, Thamer C, Peter A, Machann J, Schick F, Schraml C, et al. High cardiorespiratory fitness is an independent predictor of the reduction in liver fat during a lifestyle intervention in non-alcoholic fatty liver disease. Gut 2009;58:1281-1288.

17. Chalasani N, Younossi Z, Lavine JE, Diehl AM, Brunt EM, Cusi K, et al. The diagnosis and management of non-alcoholic fatty liver disease: practice Guideline by the American Association for the Study of Liver Diseases, American College of Gastroenterology, and the American Gastroenterological Association. Hepatology 2012;55:2005-2023.

18. Hwang I, Lee JM, Lee KB, Yoon JH, Kiefer B, Han JK, et al. Hepatic steatosis in living liver donor candidates: preoperative assessment by using breath-hold triple-echo MR imaging and $1 \mathrm{H} \mathrm{MR}$ spectroscopy. Radiology 2014;271:730-738.

19. Osawa H, Mori Y. Sonographic diagnosis of fatty liver using a histogram technique that compares liver and renal cortical echo amplitudes. J Clin Ultrasound 1996;24:25-29.

20. Matteoni CA, Younossi ZM, Gramlich T, Boparai N, Liu YC, McCullough AJ. Nonalcoholic fatty liver disease: a spectrum of clinical and pathological severity. Gastroenterology 1999;116: 1413-1419.

21. Kim SH, Lee JM, Kim JH, Kim KG, Han JK, Lee KH, et al. Appropriateness of a donor liver with respect to macrosteatosis: application of artificial neural networks to US images: initial experience. Radiology 2005;234:793-803.

22. Mancini M, Prinster A, Annuzzi G, Liuzzi R, Giacco R, Medagli $C$, et al. Sonographic hepatic-renal ratio as indicator of hepatic steatosis: comparison with (1)H magnetic resonance spectroscopy. Metabolism 2009;58:1724-1730.

23. Xia MF, Yan HM, He WY, Li XM, Li CL, Yao XZ, et al. Standardized ultrasound hepatic/renal ratio and hepatic attenuation rate to quantify liver fat content: an improvement method. Obesity (Silver 
Spring) 2012;20:444-452.

24. Schwenzer NF, Springer F, Schraml C, Stefan N, Machann J, Schick F. Non-invasive assessment and quantification of liver steatosis by ultrasound, computed tomography and magnetic resonance. J Hepatol 2009;51:433-445.

25. Sinn DH, Kang D, Chang Y, Ryu S, Gu S, Kim H, et al. Non-alcoholic fatty liver disease and progression of coronary artery calcium score: a retrospective cohort study. Gut 2017;66:323-329.

26. Lee $D H$, Lee JY, Lee KB, Han JK. Evaluation of hepatic steatosis by using acoustic structure quantification US in a rat model: comparison with pathologic examination and MR spectroscopy. Radiology 2017;285:445-453. 